\title{
Synchronization and Oscillator Death in Diffusively coupled lattice oscillators
}

\author{
Adu A.M. Wasike
}

Department of Mathematics, University of Nairobi, P.O.Box 30187, Nairobi Kenya, Email: aduwasike@yahoo.com.

\section{تززالن وموت المتنبف في المنبنبل اللشبكية المرنة لنتشاربا}

\section{ألو ولسيكي}

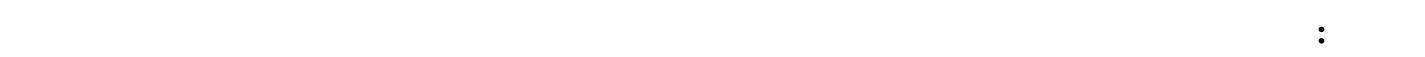

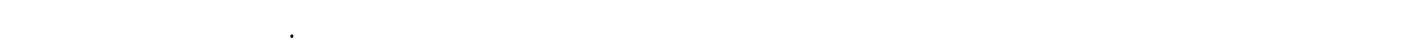

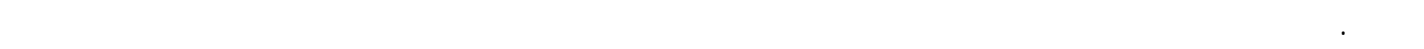

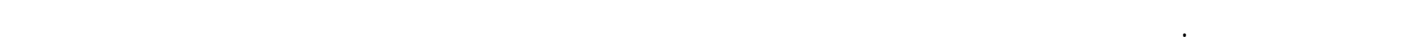
وهذا يخفي إلى الأصل بعد فارق قوي معني.
\end{abstract}

\begin{abstract}
We consider the synchronization and cessation of oscillation of a positive even number of planar oscillators that are coupled to their nearest neighbours on one, two, and three dimensional integer lattices via a linear and symmetric diffusion-like path. Each oscillator has a unique periodic solution that is attracting. We show that for certain coupling strength there are both symmetric and antisymmetric synchronization that corresponds to symmetric and antisymmetric non-constant periodic solutions respectively. Symmetric synchronization persists for all coupling strengths while the antisymmetric case exists for only weak coupling strength and disappears to the origin after a certain coupling strength.
\end{abstract}

KEYWORDS: Lattice Differential Equations, Bravais Lattice, Dynchronization and Oscillator Death.

\section{Introduction}

$\mathbf{T}$

his paper is concerned with the synchronization and mutual extinction of oscillations of a system of ordinary differential equations (ODEs) indexed by points in an $m$-dimensional integer lattice $Z^{m}, m \in\{1,2,3\}$. This system is what we shall refer to as lattice differential equations (LDEs).By a lattice oscillator, we mean a system of LDEs with every lattice points having an attracting periodic orbit.

They have been great interest in the synchronization of coupled oscillators on a onedimentional integer lattice. Aside from the mathematical interest in the problem (see for instance Aronson et. al. 1987, and references therein, coupled oscillators occur in other fields. In biology the papers of Ermentrout and Koppel $(1990,1992)$ provide us with examples where synchronization and oscillator death occur. In chemistry Bar-Eli (1985) and Crowley and Epstein (1989) consider the synchronization and oscillator death for some chemical oscillators. LDEs are also found in Cellular neural networks $(\mathrm{CNN})$, (see for example Chua and Roska 1993. Metallurgy is another area where lattice systems can be found (Cahn 1960). (private communication) has considered synchronization of coupled oscillators of subsystems that are described by a second order damped forced Duffing ordinary differential equation. The oscillators are linearly and diffusively coupled both in position and velocity to their nearest neighbours. He found that synchronization that occurred for large values of the coupling 
strength for both identical nonidentical oscillators is mainly influenced by the damping in the individual oscillators and dissipation of the coupling component of the system. Much of the work on synchronization and cessation of oscillations is done on a one-dimensional lattice and coupling involves only one variable of each subsystem. This is known in the literature as scalar coupling (Bar-Eli 1985). There is less repertoire on this subject in two and three-dimensional cases and when all variables in the subsystems at each lattice site are involved in the coupling. The problem we consider in this paper is the synchronization and cessation of oscillation of planar oscillators that are linearly and symmetrically coupled via a diffusion-like coupling to their nearest neighbours on one, two and three dimensional lattices. As a special case of the general results, we consider a canonical model where each oscillator is a truncated normal form to cubic order for a general planar oscillator near a Hopf bifurcation point. These types of planar oscillator that were considered by Poincar'e in 1891 (Perko 1998, p. 351) in his works on limit cycles, lend themselves to an easy analytical treatment. The dynamics of each oscillator $z_{j}: z_{j}(t) \in R^{2}, j=1,2,3, \ldots . .$, is governed by the solution of the equation.

$$
\dot{z}_{j}=A z_{j}-z_{j}\left|z_{j}\right|^{2}=: g\left(z_{j}\right) \text {, }
$$

where

$$
A=\left(\begin{array}{cc}
1 & -1 \\
1 & 1
\end{array}\right),
$$

|. denotes the Euclidean norm, and the "dot" denotes differentiation with respect to time $t$. The solution of Equation (1.1) for any initial condition in $R^{2} \backslash\{0$ is a unique limit cycle that attracts the whole of $R^{2} \backslash\{0\}$, see for instant (Perko 1998, page 356).

We couple the differential equations in (1.1) to yield various lattice structures. We shall first consider the dynamics of the system when coupling yields a one-dimensional lattice and then proceed to consider the two and three dimensional lattices cases.

\section{One dimensional lattice}

Now, let us couple the $\mathrm{n} \geq 2$ identical subsystems in equation (1.1) to yield

$$
z=B(k) z+f(z),
$$

where $z=\left(z_{1}, z_{2}, \ldots ., z_{n}\right)^{T}$ denotes the coordinates of a point on the lattice, $B(k)$ a real symmetric matrix depending on the coupling strength $\mathrm{k} \geq 0$, is given by $B(k)=k \Delta_{1} \otimes L$ with

$$
\Delta_{1}=\left(\begin{array}{cccccccc}
-1 & 1 & 0 & 0 & \ldots & 0 & 0 & 0 \\
1 & -2 & 1 & 0 & \ldots & 0 & 0 & 0 \\
0 & 1 & -2 & 1 & \ldots & 0 & 0 & 0 \\
: & : & : & : & \ldots & : & : & : \\
0 & 0 & 0 & 0 & \ldots & 1 & -2 & 1 \\
0 & 0 & 0 & 0 & \ldots & 0 & 1 & -1
\end{array}\right) \in R^{(n-1) \times(n-1)}, L=\left(\begin{array}{cc}
1 & 1 \\
1 & 1
\end{array}\right) ;
$$

where $\otimes$ denotes the Kronecker product, and $f(z)=\left(g\left(z_{1}\right), g\left(z_{2}\right), \ldots, g\left(z_{n}\right)\right)^{T}$. This type of coupling corresponds to a symmetric nearest neighbour coupling on a linear lattice with Neumann boundary conditions. We observe that if we had taken each subsystem in $R^{d}$ say, then the matrix $L$ above could be replaced by a $d \times d$ matrix whose entries are all 1 's. This coupling differs from the one considered in Hale (private communication) where $L$ is a $d \times d$ identify matrix. 


\section{SYNCHRONIZATION AND OSCILLATOR DEATH}

We say that Equation (2.1) is synchronized or has symmetric solutions if its solution $z$ is in the set

$$
\Theta_{1}:=\left\{z \in R^{2 n}: z_{1}=z_{2}=\ldots=z_{n} \neq 0\right\},
$$

for all $t \geq 0$. We refer to this set as the 'diagonal ' in $R^{2 n}$ (Fujisaka and Yamada 1983).

Oscillator death occur when there is a mutual cessation of oscillation in all oscillators for certain coupling strengths. That is, when $z$ is a constant for all $t \geq 0$ for some positive values of $k$. The invariant subspace

$$
\Pi_{1}:=\left\{z \in R^{2 n}: z_{j} \neq 0,1 \leq j \leq n-1\right\},
$$

defines antisymmetric solutions to equation (2.1) or antisymmetric synchronization. These two subspace are the most readily observed and are of great interest in practical problems.

The analysis of the evolution of the periodic solutions of equation (2.1) with $k$ as a parameter is facilitated by analyzing the flow restricted to the invariant manifolds $\Pi_{1}$ and $\Theta_{1}$.

Proposition 1.1. We let $n$ be an even integer. Then there exists a constant $k_{0}>0$ for which equation (2.1) defines antisymmetric solutions for all $0<k<k_{0}$. Moreover, there is oscillator death for $k>k_{0}$.

Proof. The proof involves the determinates of the eigenvalues of $B(k)$ and an appropriate change of coordinates. It is well known (Householder 1964) that the eigenvalues of $\Delta_{1}$ are

$$
\lambda_{0}=0, \lambda_{s}=-2-2 \cos \frac{s \pi}{n}, 1 \leq s \leq n-1,
$$

and they are simple. If $n$ is an even number, then clearly $\lambda_{n / 2}=-2$ is an eigenvalue and the corresponding eigenvector is $V_{n / 2}=\left(v_{1}, v_{2}, \ldots . ., v_{n}\right)^{T}$; where for a given $v_{1}= \pm I_{2}, I_{2}$ is a $2 \times 2$ identify matrix with $v_{j+1}=(-1)^{j} v_{j}, j=1,2, \ldots, n-1$. The eigenvalues of $k L$ are $\mu_{0}=0, \mu_{1}=2 k$. The set $\Pi_{1}$ is a subspace of the generalized eigenspace corresponding to the eigenvalues $-2 \mu_{\alpha}, \alpha=0,1$. We show that for any solution of equation (2.1) with an initial point in $\Pi_{1}$, there is oscillator death for all $k>k_{0}$ and antisymmetric synchronization for $k \in\left(0, k_{0}\right)$. Consider the coordinate transformation given by

$$
w_{j}=z_{j}-z_{j+1}, 1 \leq j \leq n-1, y=\frac{1}{n} \sum_{j=1}^{n} z_{j} .
$$

If we let $w:=\left(w_{1}, w_{2}, \ldots, w_{n-1}\right)^{T}$, then $w$ and $y$ satisfy the ODEs

$$
\begin{aligned}
& \dot{w}=k \Delta \otimes L w+f(w, y) \in R^{2 n-2}, \\
& \dot{y}=\frac{1}{n} \sum_{j=1}^{n} g\left(z_{j}\right) \in R^{2},
\end{aligned}
$$

where

$$
\Delta=\left(\begin{array}{cccccccc}
-2 & 1 & 0 & 0 & \ldots & 0 & 0 & 0 \\
1 & -2 & 1 & 0 & \ldots & 0 & 0 & 0 \\
0 & 1 & -2 & 1 & \ldots & 0 & 0 & 0 \\
: & : & : & : & \ldots & : & : & : \\
0 & 0 & 0 & 0 & \ldots & 1 & -2 & 1 \\
0 & 0 & 0 & 0 & \ldots & 0 & 1 & -2
\end{array}\right) \in R^{(n-1) \times(n-1)}
$$


and the function

with

$$
F(w, y)=\left(F_{1}(w, y), \ldots \ldots ., F_{n-1}(w, y)\right)^{T},
$$

$$
F_{j}(w, y)=\left(g\left(z_{j}\right)-g\left(z_{j+1}\right)\right), 1 \leq j \leq n-1 .
$$

Taking an initial condition $y_{0}$ in the manifold $\Pi_{1}$, we find that the solution of the second equation in (2.4) is $y=0$ for all $t \geq 0$ and the governing equations reduce to the decoupled system of the form

$$
\begin{gathered}
\dot{y}=0 \in R^{2}, \\
\dot{w}_{2 j-1}=-2 k l_{\frac{n}{2}} \otimes L w_{2 j-1}+g\left(w_{2 j-1}\right) \in R^{n},
\end{gathered}
$$

where $j=1,2,3, \ldots \ldots,(n / 2)$. The solution $w_{2 j-1}(t)$ of equation (2.7) corresponds to antisymmetric solutions of the system (2.1). To show the existence of $k_{0}$, we need consider the case for $n=2$ let only as the system equation (2.7) is decoupled and the result for $n>2$ will follow easily. Let

$$
\alpha=\alpha(k)=\frac{2 k}{\sqrt{(1-2 k)^{2}+1}}, \tan 2 \phi=1-2 k .
$$

We introduce a change of coordinates in equation (2.7) with $n=2$ given by

$$
\left(\begin{array}{l}
U \\
V
\end{array}\right)=\left(\begin{array}{cc}
\sqrt{1+\alpha} & 0 \\
0 & \sqrt{1-\alpha}
\end{array}\right)\left(\begin{array}{cc}
\sin \phi & \cos \phi \\
\cos \phi & \sin \phi
\end{array}\right) w_{1} .
$$

The equation (2.7) becomes

$$
\begin{aligned}
& \left(\begin{array}{l}
U^{\prime} \\
V^{\prime}
\end{array}\right)=\left\{\begin{array}{cc}
1-2 k-2 k \sin 2 \phi & (1-2 k \cos 2 \phi) \sqrt{\frac{(1+\alpha)}{(1-\alpha)}} \\
-(1+2 k \cos 2 \phi) \sqrt{\frac{(1-\alpha)}{(1+\alpha)}} & 1-2 k+2 k \sin 2 \phi
\end{array}\right) \\
& \left.-\left(\frac{U^{2}+V^{2}-\alpha\left(U^{2}-V^{2}\right)}{1-\alpha^{2}}\right) I_{2}\right\}\left(\frac{U}{V}\right) .
\end{aligned}
$$

in which the prime denotes differentiation with respect to time. In polar coordinates defines by

equation (2.9) becomes

$$
U=p(t) \cos \sigma(t), V=p(t) \sin \sigma(t),
$$

$$
\begin{aligned}
& p^{\prime}=(1-\alpha \cos 2 \sigma)\left(1-2 k-\frac{p^{2}}{1-\alpha^{2}}\right) p, \\
& \sigma^{\prime}=-\sqrt{1-\alpha^{2}}+(1-2 k) \alpha \sin 2 \sigma .
\end{aligned}
$$

Solving $p^{\prime}=0$ for $p$ in the first equation in (2.10), one finds that

$$
p^{*}=\sqrt{(1-2 k)\left(1-\alpha^{2}\right)}
$$

provided $1>2 k$ and $\alpha<1$, is the radius of a unique invariant circle. $\sigma^{\prime}<0$ when 


\section{SYNCHRONIZATION AND OSCILLATOR DEATH}

$0 \leq k<1 / 2$ and

$$
P^{\prime}\left\{\begin{array}{lll}
<0, & \text { When } & p>P^{*} \\
>0, & \text { When } & p<P^{*}
\end{array}\right.
$$

Therefore $P^{*}$ is the underlying point set if a limit cycle of $(2.10)$ and attracts all of $R^{2} \backslash(0,0)$ as $t \rightarrow \infty$ that exists when $0 \leq k<1 / 2$. If we let $w_{1}=(r(t) \cos \theta(t), r(t))$, then it can be shown that equation (2.7) with $0<k<1 / 2$, admits a unique and asymptotically stable periodic orbit with period $T(\alpha)$ and radius $r(\alpha)$ given by

$$
T(\alpha)=\frac{2 \pi}{\sqrt{1-(2 \alpha)^{2}}}, r(\alpha) \sqrt{\frac{(1-2 \alpha)\left(1-\alpha^{2}\right)}{1-\alpha \cos 2(\theta(t)+\phi)}} .
$$

Clearly at $k_{0}=k=1 / 2, r=0$; i.e. there is no oscillation. Simple analysis shows that for $k>k_{0}$, the origin for system (2.7) is stable.

\section{Two-dimensional lattice}

We consider an $n \times n$ simple (or Bravais) square lattice. Let $z_{i j}, 1 \leq i, j \leq n$, be coordinate of the $(i, j)^{\text {th }}$ site oscillator and the matrix $Z:=\left[z_{i j}\right]_{i, j=1}$. Let $Z=\left(Z_{1}, Z, \ldots \ldots Z_{n}\right)$ with $Z_{r} \in R^{2 n \times 1}$, $1 \leq r \leq n$, as its $r$ th column. Let vec $Z$ denote the vector valued function of $Z$, and $\otimes$ be the Kronecker sum (See Graham 1981, for details on matrix tensor algebra).

The system of differential equations describing the dynamics on the $n \times n$ lattice is given by

$$
\dot{\boldsymbol{Z}}=H(k) \boldsymbol{Z}+G(\boldsymbol{Z})
$$

where $H(k)=k\left[\left(\Delta_{1} \otimes \Delta_{1}\right) \otimes L\right]$, and $G(\boldsymbol{Z}):=\left[g\left(z_{i j}\right)\right]_{i, j=1}^{n}=\left(f\left(Z_{1}\right), f\left(Z_{2}\right), \ldots ., f\left(Z_{n}\right)\right)$.

Equation (3.1) is symmetrically synchronization when $z_{i j}=z \neq 0$ for all $i j$. This occurs when $H(k) \boldsymbol{Z}=\mu \boldsymbol{Z}=0, Z \neq 0$ with $\mu=0$ as an eigenvalue of $H(k)$. Indeed, the eigenvalues of $H(k)$ are

$$
\sigma(H(k)):=\left\{\mu: \mu=\left(\lambda_{s}+\lambda_{p}\right) \mu_{\alpha}, 0 \leq s, p \leq n-1\right\},
$$

with $\lambda_{p}, \lambda_{s}, \mu_{\alpha}, \alpha=0,1$, as defined in section 2. Clearly $\mu=0$ is an eigenvalue of $H(k)$ and by equation (3.1), there is symmetric synchronization. In this case, the plane will be ramified with traveling-wave like pattern solutions to equation (3.1).

To facilities the understanding of other dynamics, we define some subspaces invariant to the flow defined by equation (3.1). Here, we shall follow a trend similar to that in Mallet-Paret and Chow (1995a, 1995b) where pattern formations are considered for LDE's with a first order scalar ODE at each lattice site. Let

$$
\begin{aligned}
& \Theta_{\theta \theta}=\left\{Z: Z_{j+1}=Z_{j}, Z_{j} \in \Theta_{1}, 1 \leq j \leq n-1\right\}, \\
& \prod_{\pi \theta}=\left\{Z: Z_{j+1}=(-1)^{j} Z_{j} \in \Theta_{1}, 1 \leq j \leq n-1\right\}, \\
& \prod_{\pi \pi}=\left\{Z: Z_{j+1}=(-1)^{j} Z_{j}, Z_{j} \in \prod_{1}, 1 \leq j \leq n-1\right\}, \\
& \prod_{\pi \theta}=\left\{Z: Z^{T} \in \prod_{\pi \theta}\right\} .
\end{aligned}
$$

We further make a coordinate transformation defined thus 


$$
W_{j}=Z_{j}-Z_{j+1}, 1 \leq j n-1, \text { and } Y=\frac{1}{n} \sum_{j=1}^{n} Z_{j} .
$$

Using this in equation (3.1) we get :

$$
\begin{aligned}
& \dot{W}=k\left[\left(I_{n-1} \otimes L\right) W \Delta+\left(\Delta_{1} \otimes L\right) W I_{n-1}\right]+\tilde{G}(W, Y) \in R^{2 n(n-1)} \\
& \dot{Y}=B(k) Y+\frac{1}{n} \sum_{j=1}^{n} f\left(Z_{j}\right) \in R^{2 n},
\end{aligned}
$$

where

$$
\begin{gathered}
W=\left(W_{1}, \ldots, W_{n-1}\right), \\
\tilde{G}(W, Y)=\left(\tilde{G}_{1}(W, Y), \ldots ., \tilde{G}_{n-1}(W, Y)\right),
\end{gathered}
$$

with

$$
\tilde{G}(W, Y)=f\left(Z_{j}\right)-f\left(Z_{j+1}\right), 1 \leq j \leq n-1 .
$$

on the subspace $\Theta_{\theta \theta}$

$$
\begin{aligned}
& \dot{W}=0 \in R^{2 n(n-1)}, \\
& \dot{Y}=\dot{Z}_{\beta}=f\left(Z_{\beta}\right) \in R^{2 n}, \beta \in\{1,2, \ldots \ldots, n\} .
\end{aligned}
$$

The system in this case is symmetrically synchronized and the dynamics are described by equation (3.4).

Proposition 2.1. Let $n$ be an even integer. Then there exists a constant $k_{1}>0$ for which the simple lattice equation (3.1) is ramified with horizontal and vertical bands of antisymmetric solutions for all $k \in\left(0, k_{1}\right)$. Moreover, oscillator death occurs for $k>k_{1}$.

Proof. On $\prod_{\theta \pi}$ and $\prod_{\pi \theta}$, we have horizontal and vertical bands of stripes respectively and their flows are governed by the decoupled ODEs,

$$
\begin{aligned}
& \dot{Y}=0, \\
& W_{2 j-1}=\left[I_{\frac{n}{2}} \otimes\left(B(k)-2 k I_{n} \otimes L\right)\right] W_{2 j-1}+f\left(W_{2 j-1}\right),
\end{aligned}
$$

where $W_{2 j+1}=(-1)^{j} W_{2 j-1}$, with $j=1,2,3, \ldots ., n / 2$. Since all $Z_{j} \in \Theta_{1}$, equation (3.5) reduces to $n / 2$ decoupled ODEs. Thus

$$
\dot{W}_{2 j+1}=\left(-2 k I_{n} \otimes L\right) W_{2 j-1}+f\left(W_{2 j-1}\right)
$$

which upon the use of equation (2.7), Proposition 2.1 is proved with $k_{1}=1 / 2$.

Proposition 2.2. Let $n$ be an even integer. Then there is a constant $k_{2}>0$ for which the lattice equation (3.1) is ramified with checkerboard like solutions for all $k \in\left(0, k_{2}\right)$. Moreover, oscillator death occurs for $k>k_{2}$.

Poof. The subspace $\prod_{\pi \pi}$ represents vertical (horizontal) bands of symmetric and antisymmetric oscillators. This subspace constituted a checkerboard. A unit square on a checkerboard is constituted by four subsystems on a $2 \times 2$ square lattice. Within each square, the subsystems are of alternate signs with their nearest neighbours. If $z(t)$ represents an oscillator, then 


\section{SYNCHRONIZATION AND OSCILLATOR DEATH}

$$
\left(\begin{array}{cc}
z(t) & -z(t) \\
-z(t) & z(t)
\end{array}\right)
$$

is the unit square. The entire checkerboard is defined by these squares that alternate is sign. The subspace $\prod_{\pi \pi}$ is invariant under the flow defined by equation (3.1) and its flow governed by the solution of the $n / 2$ equations

$$
\dot{W}_{2 j-1}=(-4 k I \otimes L) W_{2 j-1}+f\left(W_{2 j-1}\right)
$$

We notice that equation (3.7) is similar to equation (2.7). The proof of the Proposition 2.2 follows easily if we replace $k$ in equation (2.7) with $2 k$. We find that $k_{2}=1 / 4$.

\section{Three dimensional lattice}

We consider an $n \times n \times n$ simple cubic lattice. Let $z_{p q r}, 1 \leq p, q, r \leq n$, be the coordinate of the $(p, q, r)^{t h}$ site oscillator with its dynamics described by equation (1.1). We shall assume that the coupling is to the nearest neighbour and the coupling forces are equal and acting along the axes of

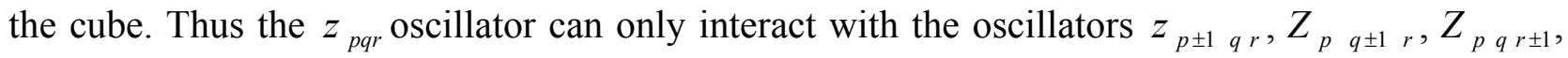
six in all. Let $\mathbf{Z}:=\left(\mathbf{Z}_{1}, \mathbf{Z}_{2}, \ldots . ., \mathbf{Z}_{n}\right)^{T}$ where $\mathbf{Z}_{r}:=\left[z_{p q r}\right]_{p, q, r=1}^{n}$. The system of differential equations describing the dynamics of the entire lattice system is given by

$$
\dot{\mathbf{Z}}=\mathbf{H}(k) \mathbf{Z}+\mathbf{G}(\mathbf{Z}) \text {, }
$$

where

$$
\mathbf{H}(k)=k\left[\left(\left(\Delta_{1} \otimes \Delta_{1}\right) \otimes\left(I_{n} \otimes \Delta_{1}\right)\right) \otimes L\right], \mathbf{G}(\mathbf{Z})=\left(G\left(\mathbf{Z}_{1}\right), \ldots, G\left(\mathbf{Z}_{n}\right)\right)^{T},
$$

with

$$
G\left(\mathbf{Z}_{r}\right)=\left[g\left(z_{p q r}\right)\right]_{p, q, r=1}^{n} .
$$

To determine the invariant subspaces available to equation (4.1), we find the eigenvalues of $\mathbf{H}(k)$ and the corresponding eigenvectors. We have

$$
\sigma(\mathbf{H}(k)):=\left\{\mu: \mu=\left(\left(\lambda_{p}+\lambda_{q}\right)+\lambda_{r}\right) \mu_{\alpha}, 0 \leq p, q, r \leq n-1\right\},
$$

where $\lambda_{p}, \lambda_{q}, \lambda_{r}, \mu_{\alpha}$, are as defined in section 2 with each $\lambda_{r}$ occurring with multiplicity $n$.

$$
\begin{aligned}
& \Theta_{\theta \theta \theta}=\left\{\mathbf{Z}: \mathbf{Z}_{j+1}=\mathbf{Z}_{j}, \mathbf{Z}_{j} \in \Theta_{\theta \theta}, 1 \leq j \leq n-1\right\}, \\
& \prod_{\pi \pi \theta}=\left\{\mathbf{Z}: \mathbf{Z}_{j+1}=\mathbf{Z}_{j}, \mathbf{Z}_{j} \in \prod_{\pi \pi}, 1 \leq j \leq n-1\right\}, \\
& \prod_{\theta \pi \theta}=\left\{\mathbf{Z}: \mathbf{Z}_{j+1}=\mathbf{Z}_{j}, \mathbf{Z}_{j} \in \prod_{\theta \pi}, 1 \leq j \leq n-1\right\}, \\
& \Theta_{\theta \theta \pi}=\left\{\mathbf{Z}: \mathbf{Z}_{j+1}=(-1)^{j} \mathbf{Z}_{j}, \mathbf{Z}_{j} \in \Theta_{\theta \theta}, 1 \leq j \leq n-1\right\}, \\
& \prod_{\theta \pi \pi}=\left\{\mathbf{Z}: \mathbf{Z}_{j+1}=(-1)^{j} \mathbf{Z}_{j}, \mathbf{Z}_{j} \in \prod_{\theta \pi}, 1 \leq j \leq n-1\right\}, \\
& \prod_{\pi \pi \pi}=\left\{\mathbf{Z}: \mathbf{Z}_{j+1}=(-1)^{j} \mathbf{Z}_{j}, \mathbf{Z}_{j} \in \prod_{\pi \pi}, 1 \leq j \leq n-1\right\}, \\
& \prod_{000}=\{\mathbf{Z}: \mathbf{Z}=0\} .
\end{aligned}
$$

The system of equations defining the dynamics on these manifolds follows readily with the coordinate transformation

$$
\begin{aligned}
& \dot{\mathbf{W}}_{j}: \mathbf{Z}_{j}-\mathbf{Z}_{j+1}, \quad 1 \leq j \leq n-1, \\
& \mathbf{Y}:=\frac{1}{n} \sum_{j=1}^{n} \mathbf{Z}_{j} .
\end{aligned}
$$

Using equation (4.2 in equation (4.1), we get 


$$
\begin{aligned}
& \dot{\mathbf{W}}: k\left[\left(\left(\Delta \otimes I_{n}\right) \otimes\left(\Delta_{1} \otimes \Delta_{1}\right)\right) \otimes L\right] \mathbf{W}+\tilde{\mathbf{G}}(\mathbf{W}, \mathbf{Y}), \\
& \dot{\mathbf{Y}}=H(k) \mathbf{Y}+\frac{1}{n} \sum_{j=1}^{n} G\left(\mathbf{Z}_{j}\right),
\end{aligned}
$$

where

$$
\mathbf{W}=\left(\mathbf{W}_{1}, \ldots ., \mathbf{W}_{n-1}\right)^{T}, \tilde{\mathbf{G}}(\mathbf{W}, \mathbf{Y})=\left(\tilde{G}_{1}(\mathbf{W}, \mathbf{Y}), \ldots \ldots, G_{n-1}(\mathbf{W}, \mathbf{Y})\right)^{T},
$$

with $\tilde{\mathbf{G}}(\mathbf{W}, \mathbf{Y})=G\left(\mathbf{Z}_{j}\right)-G\left(\mathbf{Z}_{j+1}\right), 1 \leq j \leq n-1$, on $\Theta_{\theta \theta \theta}, \mathbf{W}=\mathbf{0}$, and the flow is governed by

$$
\dot{\mathbf{Z}}_{\beta}=G\left(\mathbf{Z}_{\beta}\right), \beta=1, \ldots, n .
$$

We thus see that the system is synchronized

Proposition 3.1. Let $n$ be an even integer. Then for all $k \in\left(0, k_{3}\right)$ the simple three-dimensional lattice equation (4.1) has layers of checkerboards. Besides, oscillator death occurs for $k>k_{3}$. Proof. On the subspace $\prod_{\pi \pi \theta}$, the flow is defined by the solution of the decoupled equation

$$
\dot{\mathbf{Z}}_{\beta_{j}}=-4 k\left(I_{n} \otimes L\right) \mathbf{Z}_{\beta_{j}}+f\left(\mathbf{Z}_{\beta_{j}}\right), \beta, j=1, \ldots, n,
$$

where $\mathbf{Z}_{\beta_{j}}$ is the $\mathrm{j}^{\text {th }}$ column of $\mathbf{Z}_{\beta}$. Thus we shall have layers of checkerboards that persist for $0<k<1 / 4$. Notice the similarity of equation (4.5) with equation (2.7). Thus replacing $k$ in equation (2.7) with $2 k$ we find that $k_{3}=1 / 4$.

Proposition 3.2. Let $n$ be an even integer. Then there exists a constant $k_{4}>0$ such that for all $k \in\left(0, k_{4}\right)$ the simple three-dimensional lattice equation (4.1), has sheets with vertical bands of stripes of checkerboards. Moreover, oscillator death occurs for $k>k_{4}$.

Proof. On the subspace $\prod_{\pi \theta \theta}$, the flow is defined by the solution of the decoupled equation

$$
\mathbf{Z}_{\beta_{j}}=-2 k\left(I_{n} \otimes L\right) \mathbf{Z}_{\beta_{j}}+f\left(\mathbf{Z}_{\beta_{j}}\right), \beta, j=1, \ldots, n,
$$

where $\mathbf{Z}_{\beta_{j}}$ is the $\mathrm{j}^{\text {th }}$ column of $\mathbf{Z}_{\beta}$. Thus the entire system in the three-dimensional space is composed of sheets with vertical bands of stripes. Clearly, we see from equation (2.7) that this regime persists for $0<k<1 / n$. Proposition 3.2 follows with $k_{4}=1 / 2$.

On $\prod_{\theta \pi \theta}$, the flow is defined by the solution of the $n^{3}$ decoupled equations in (4.6). The solutions represent layers of sheets with horizontal band strips that persists for $0<k<1 / 2$.

Proposition 3.3. Let $n$ be an even integer. Then there exists a constant $k_{5}>0$ for which the simple three-dimensional lattice equation (4.1) has layers of horizontal sheets with horizontal bands that persist for all $k \in\left(0, k_{5}\right)$. Moreover, oscillator death occurs for $k>k_{5}$.

Proof. On the subspace $\Theta_{\pi \theta \theta}$ the flow is defined by the solution of the decoupled equations

$$
\dot{\mathbf{W}}_{2 j-1}=-2 k\left(I_{n} \otimes L\right) \mathbf{W}_{2 j-1}+\tilde{G}\left(\mathbf{W}_{2 j-1}\right) j=1,2, \ldots, n / 2 \text {. }
$$

Thus we shall have horizontal bands of synchronized sheets, like a "stack of sandwiches" that persists for $0<k<1 / 2$. Proposition 1.1 follows with $k_{5}=1 / 2$.

On $\prod_{\theta \pi \pi}$, the flow is governed by equation (4.7) and we have sheets with horizontal bands.

Proposition 3.4. Let $n$ be an even integer, then there exists a constant $k_{\sigma}>0$ for which the simple three-dimensional lattice equation (4.1), has layers of horizontal sheets of checkerboards that persist for all $k \in\left(0, k_{\sigma}\right)$. Moreover, oscillator deaths occur for $k>k_{\sigma}$.

Proof. On the subspace $\prod_{\pi \pi \pi}$, the flow is defined by the solution of the decoupled equations

$$
\dot{\mathbf{W}}_{2 j-1}=-4 k\left(I_{n} \otimes L\right) \mathbf{W}_{2 j-1}+\tilde{G}\left(\mathbf{W}_{2 j-1}\right), j=1,2, \ldots, n / 2
$$




\section{SYNCHRONIZATION AND OSCILLATOR DEATH}

Thus the entire system in three-dimensional space is composed of layers of horizontal sheets of checkerboard. This dynamical regime persists for $0<k<1 / 4$. Proposition 3.4 follows with $k_{6}=1 / 4$.

\section{Discussion}

In LDE's on the Bravais lattices given in equations (2.1),(3.1) and (4.1) the coupling matrices $B(k), H(k),\{H\}(k)$ have $\lambda_{0}=0$ as an eigenvalue. Thus any solution to these equations that pass through a point in the generalized eigenspace corresponding $\mu_{i} \lambda_{0}^{v}, v=1,2,3$, respectively, shall be symmetrically synchronized. That is, they shall be in the set $\Theta_{1}, \Theta_{\theta \theta}, \Theta_{\theta \theta \theta}$, respectively. For an even number of oscillators in the bravais lattice, the coupling matrices have $\lambda_{n / 2}=-2$ as an eigenvalue. From equations (2.7),(3.7),(4.5), we see that any solution to equation (2.1),(3.1),(4.1) through a point in the generalized eigenspace belonging to $\lambda_{n / 2}=-2$ will always be antisymmetric for some coupling strength $k: 0<k \leq \tilde{k}$ and there shall be oscillator death for $k \geq \tilde{k}$. Thus simple Bravais osillators with each osillator as given in equation (1.1) can either lead to synchronization or oscillator death depending on the initial conditions and the strength of coupling.

\section{References}

ARONSON, D.G., DOEDEL, E.J., and OTHMER, H.G. 1987. An analytical amd numerical study of the bifurcations in a system of linearly coupled oscillators. Physica D 25: 20-104.

BAR-ELL, K. 1985. On the stability of coupled chemical oscillators. Physica D. 14: 242-252.

CHN, J.W. 1960. Theory of growth and interface motion in crystalline material, Acta Metallurgica 8: $554-562$.

CHUA, L.O, and ROSKA, T. 1993. The CNN Paradigm. IEEE Trans. Circuits Syst., 40: 147-156.

M.F. CROWLEY, M.F., EPSTEIN, I. 1989. Experimental and theoretical studies of a coupled chemical oscillator : Phase Death, Multistability, and in-Phase and Out of phase and Entrainment. J. Phys. Chem. 93: 2496-2502.

ERMENTOUT, G.B. KOPPEL, N. 1994 Inhibition-produced patterning in chains of coupled nonlinear oscillators. SIAM J. Appl. Maths. 54: 478-507.

H. FUJISAKA, H. and YAMADA, H. 1983. Stability Theory of Synchronized Motion in coupledOscillator Systems Prog. Theor. Phys. 69: 32-47.

GRAHAM, A. 1981. Kronecker Products and Matrix Calculus with Applications, Ellis Horwood Limited.

HOUSEHOLDER. A.S. 1964. The Theory of Matrices in Numerical Analysis. Blaisdell.

MALLET-PARET, J. and CHOW, S.-N. 1995. Pattern formation and spatial chaos in lattice dynamical systems II. IEEE Trans. Circuits Syst. 42: 752-756.

PERKO, L. 1998. Differential Equation and dynamical system 2nd. Ed. Springer-Verlag, Newyork, Berlin Heidelberg. 19.

POINCACARE, H. 1891 Memoire sur les courbes dfnites par une equation differentielle,. $J$. Mathematiques, 7: 375-421.

Received 11 June 2002

Accepted 28 April 2003 